\title{
A Hierarchical Coevolutionary Method to Support Brain-Lesion Modelling
}

\author{
Michail Maniadakis and Panos Trahanias \\ Institute of Computer Science, Foundation for Research and Technology-Hellas (FORTH), 71110 Heraklion, Crete, Greece \\ and \\ Department of Computer Science, University of Crete, 71409 Heraklion, Crete, Greece \\ E-mail: $\{$ mmaniada, trahania\}@ics.forth.gr
}

\begin{abstract}
The current work addresses the development of cognitive abilities in artificial organisms, a topic that has attracted many research efforts recently. In our approach, neural networkbased agent structures are employed to represent distinct brain areas. We introduce a Hierarchical Collaborative CoEvolutionary (HCCE) approach to design autonomous, yet cooperating agents. Thus, partial brain models consisting of many substructures can be designed. Replication of lesion studies is used as a means to increase reliability of brain model, highlighting the distinct roles of agents. The HCCE is appropriately designed to support systematic modelling of brain structures, able to reproduce biological lesion data. The proposed approach designs cooperating agents properly, by considering the desired pre- and post- lesion performance of the model. The effectiveness of the proposed approach is illustrated on the design of a computational model of Primary Motor cortex and Premotor cortex interactions in the mammalian brain. The model is successfully tested in driving a simulated robot, with different pre- and post- lesion performance.
\end{abstract}

\section{INTRODUCTION}

Cognitive abilities of animals are supported by the performance of their Central Nervous System (CNS). The latter consists of several interconnected modules with different functionalities [1]. A lot of research is recently oriented towards determining how these modules cooperate to accomplish real world tasks [2]. Even if the detailed, exact properties of each brain area are not clear yet, many computational models have been proposed capturing their basic characteristics [3], [4], [5], [6]. Recently, computational studies investigate the performance of the models in lesion conditions as a means to increase their reliability [7], [8], [9].

We have recently introduced a systematic method to design biologically plausible computational models of partial CNS structures [10], [11]. In accordance to the distributed organization of the mammalian CNS, an agent-based modelling approach is followed. Specifically, the model consists of a collection of neural agents, each one representing a CNS area. The agent-based approach enforces the autonomy of brain areas, supporting also problem decomposition in small tractable tasks.

The performance of agents is specified by means of environmental interaction similar to an epigenetic ${ }^{1}$ learning process. The dynamics of epigenetic learning are designed

\footnotetext{
${ }^{1}$ Epigenesis here, includes all learning processes during lifetime.
}

by an evolutionary process which simulates phylogenesis, similar to [12], [13]. Consequently, both genetically encoded features and subjective experience have a significant role in the formation of model's performance. Following the phylogenetic/epigenetic approach, the objective adopted during the evolution of agents, is to furnish them with abilities to develop similar performance to the respective brain areas, after a certain amount of environmental interaction.

Instead of using a unimodal evolutionary process we employ a cooperative coevolutionary approach which is able to highlight the specialties of brain areas represented by distinct agents [10]. Additionally, the coevolutionary approach facilitates the integrated performance of substructures in the composite model. The combination of these two particular features (partial autonomy and collaborative performance) in a single design method seems particularly appropriate for brain modelling.

In the present work, we propose a hierarchical extension of this approach, which exploits the inherent ability of coevolutionary methods to integrate partial structures. We introduce a Hierarchical Colaborative CoEvolutionary (HCCE) scheme which supports the coevolution of a large number of species (populations). Specifically, evolutionary processes at lower levels are driven by their own dynamics to fulfill the special objectives of each brain area. The evolutionary process at the higher levels, tunes lower level coevolutionary process to achieve the integrated performance of partial structures. The architecture of multiple coevolutionary processes tuned by a higher level evolution can be repeated for as many levels as necessary, forming a tree hierarchy.

It should be noted that the composite model does not have to perform in a hierarchical mode. The performance of partial CNS structures can be either hierarchical or completely parallel, depending on the biological prototype. Hence, the hierarchical coevolutionary approach does not imply any further constraints. It is introduced only to support the design process of brain modelling.

Furthermore, following recent trends aiming at the study of computational models in lesion conditions [7], [8], [9], we adapt our method to accomplish systematic modelling of biological lesion experiments. The agent-based representation of brain areas facilitates lesion simulation by simply deactivating appropriate agent structures. Thus, the performance of 
the model in pre- and post- lesion conditions can be tested. Furthermore, appropriate fitness functions can be specified for the evolution of partial structures, to indicate the performance of the model when all substructures are present, and also indicate the performance when some partial structures are eliminated. Following this approach, biological lesion data can be considered during the coevolutionary design process and computational structures are properly formulated to replicate pre- and post- lesion performance. Consequently, increased reliability is offered to the final model.

The rest of the paper is organized as follows. In the next section, we present the basic characteristics of the neural agent structures employed for the representation of CNS areas, and the hierarchical collaborative coevolutionary scheme which supports agents' design. The results of the proposed approach on a brain modelling task are presented in section III. Specifically we demonstrate the design of a computational model of primary motor cortex - premotor cortex interactions in the mammalian brain. The model is embedded in a robotic platform, which supports environmental interaction. When both cortical agents are active the robot is able to achieve goal oriented purposeful motion, while when the agent of premotor cortex is eliminated it can only achieve a wall avoidance behavior. Finally, conclusions and suggestions for future work are drawn in the last section.

\section{METHOD}

Modern theories for the explanation of mammalian cognition argue that the observed behavior of animals is a result of phylogenetic development, and epigenetic environmental experience [14]. Evidently, this argument may also form a basis to accomplish brain modelling tasks [12], [13]. An evolutionary method can be employed to specify the dynamics of real-time learning process.

\section{A. Computational Model}

We have implemented two different neural network based agents, to supply general computational structures for brain modelling: (a) a computational cortical agent to represent brain areas, and (b) a link agent to support information flow across brain areas. Thus, an appropriately complex connectivity can be defined, to simulate connectivity of CNS modules.

We note that the proposed computational model is not restrictive for the coevolutionary method, but rather serve as a guide on how coevolutionary approaches can be employed to support brain modelling tasks. Additional constraints can be integrated to increase its biological reliability. The computational details of cortical and link agents have been presented elsewhere [6], [10], [11]. In the present document, due to space limitations, we will summarize only their basic characteristics.

1) Cortical Agent: Each cortical agent consists of a population of excitatory and inhibitory neurons, following the Wilson-Cowan model similar to [15]. A rectangular plane simulates cortical area. Both sets of neurons are uniformly distributed in the cortical plane. Four sets of intra-cortical

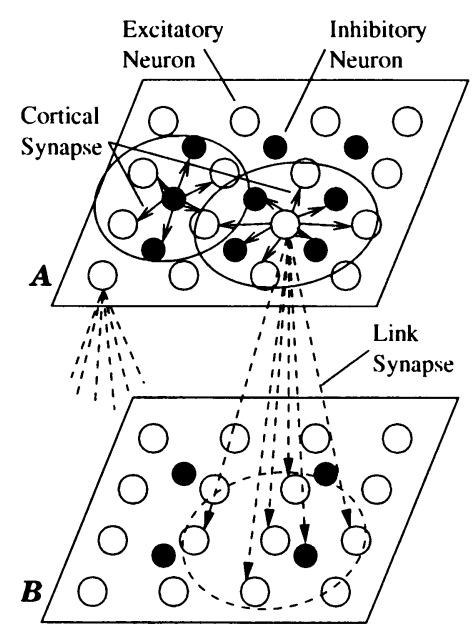

(a)

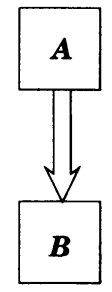

Fig. 1. A schematic representation of the agents employed to represent brain areas. Part (a) illustrates the artificial neural structure of cortical and link agents. Intra-cortical synapses and neighborhoods are illustrated with solid lines. Inter-cortical synapses and neighborhoods are illustrated with dashed lines. Part (b) illustrates the compact representation of agents connectivity which is followed throughout the paper. Cortical agents are illustrated with blocks, while link agents are illustrated with double arrows.

synapses are defined depending on the nature of presynaptic and postsynaptic neurons (excitatory-excitatory, excitatoryinhibitory, inhibitory-excitatory, inhibitory-inhibitory). The connectivity of neurons follows the general rule of locality. Synapse formation in cortical agents is based on circular neighborhoods. This is demonstrated graphically in Fig 1.

2) Link Agent: An appropriate link agent is specified to allow information flow across cortical agents. Inter-cortical synapses of link agents are specified based on the spatial properties inherited by the planar model of cortical agents. Synapse definition follows the principle that neighboring cells project to neighboring areas. Thus, a circular neighborhood measure is also employed to specify locality across cortical agents. Inter-cortical locality is approximated by the circular neighborhoods defined after projecting the neurons of the linked cortical agents on a common virtual plane (Fig 1). Only excitatory neurons are used as outputs of the efferent cortical module, while both excitatory and inhibitory neurons receive input in the afferent module. Thus, two sets of synapses are specified for each link agent (excitatory-excitatory, excitatoryinhibitory). Using the link structure any two cortical agents can be connected. As a result, the connectivity of CNS modules can be easily simulated.

3) Epigenetic Learning: Experience based subjective learning has an important contribution to the final performance of brain models. To enforce epigenetic learning, each set of synapses (for both link and cortical agents) is assigned a Hebbian-like learning rule to enforce agents' self-organization, similar to [16]. The assignment of the proper learning rule in each synapse set allows the emergence of the desired performance in agent structures, after a certain amount of environmental interaction. We have implemented a pool of 
10 Hebbian-like rules that can be appropriately combined to produce a wide range of functionalities during lifetime adjustment.

\section{B. Hierarchical Collaborative CoEvolution (HCCE)}

Similar to a phylogenetic process the specification of parameter values for all agents is approached in a systematic way by using an evolutionary mechanism, as it has been suggested in [12], [13]. Furthermore, coevolutionary algorithms have been recently proposed that facilitate exploration, in problems consisting of many decomposable subcomponents [17]. They involve two or more coevolved species (populations) with interactive performance. The brain modelling problem fits very well to collaborative coevolutionary approaches, because separate coevolved species can be used to perform design decisions for each partial model of a brain area, enforcing both a performance similar to reality and the cooperation within computational brain modules.

We have presented a new evolutionary scheme to improve the performance of collaborative coevolutionary algorithms, by explicitly addressing the collaborator selection issue [10], [11]. The present work extents this scheme to a hierarchical multi-level architecture. Our method combines the hierarchical evolutionary approach [18], with the maintenance of successful collaborator assemblies [19], to develop a powerful coevolutionary scheme.

We employ two different kinds of species (populations) to support the coevolutionary process encoding the configurations of either a Primitive agent Structure (PS) or a Coevolved agent Group (CG). PS species specify partial elements of the model, encoding the exact structure of either cortical or link agents. A CG consists of groups of PSs with common objectives. Thus, CGs specify configurations of partial solutions by encoding individual assemblies of cortical and link agents. The evolution of CG modulates partly the evolutionary process of its lower level PS species to enforce their cooperative performance. A CG can also be a member of another CG. Consequently several CGs can be organized hierarchically in a tree-like architecture, with the higher levels enforcing the cooperation of the lower ones.

The HCCE-based design method for brain modelling is demonstrated by means of an example (Fig 2). We assume the existence of two cortical agents connected by three link agents representing their afferent and efferent projections (Fig 2(a)). One hypothetical HCCE process employed to specify agent structure is illustrated in (Fig 2(b)).

Similar to [18], [10] all individuals in all species are assigned an identification number which is preserved during the coevolutionary process. The identification number is employed to form individual assemblies within different species. Each assembly specifies a complete problem solution which is further tested on the desired task.

Each variable in the genome of a CG is joined with one lower level CG or PS species. The value of that variable can be any identification number of the individuals from the species it is joined with. PSs encode the structure of either cortical or

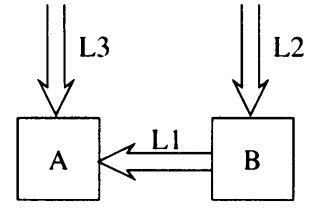

(a)

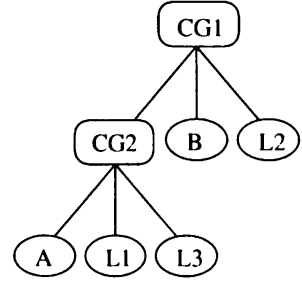

(b)
Fig. 2. Hierarchical collaborative coevolutionary design of agents. Part (a) represents schematically a hypothetical connectivity of agents. Part (b) represents the hierarchical coevolutionary scheme utilized to evolve partial structures. CGs are illustrated with oval boxes, while PSs are represented by ovals.

link agents. The details of the encoding have been presented in [10], [11], and thus they are omitted here due to space limitations. CGs enforce cooperation of PS structures by selecting the appropriate cooperable individuals among species. Additionally, a new genetic operator, termed Replication [10], exploits the most able to cooperate individuals in each partial species. A snapshot of the exemplar HCCE process described above is illustrated in (Fig 3).

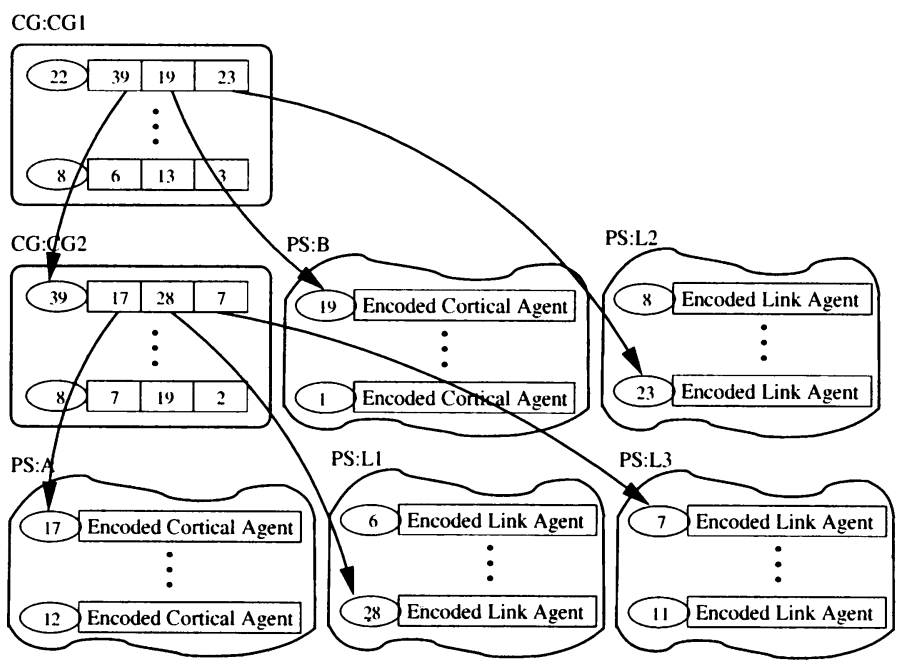

Fig. 3. An overview of the hierarchical coevolutionary scheme, with CG species tuning the evolutionary processes of PS species. Identification numbers are represented with an oval.

In order to test the performance of a complete problem solution, populations are sequentially accessed starting by the higher level. The values of CG individuals at various levels are used as guides to select collaborators among PS species. Then, PS individuals are decoded to specify the structure of cortical and link agents, and the performance of the proposed overall solution is tested on the desired task.

The hierarchical organization is able to simulate lesion studies by deactivating appropriate nodes of the tree hierarchy. Deactivated nodes correspond to lesioned structures of the composite model. Consequently, all lesion conditions can be considered. Furthermore, the coevolutionary process can utilize appropriately formulated fitness functions to specify the 
desired pre- and post- lesion performance of the model. As a result, the proposed architecture is able to reproduce data obtained by biological lesion studies.

Evolutionary processes at both CG and PS species are driven by their own fitness functions. This is particularly important for agents' coevolution since different objectives can be defined for each agent, thus preserving their autonomy. Especially for a brain modelling application, separate fitnesses are able to highlight the special features of partial agents representing distinct brain areas.

An evaluation index represents how good is the solution formed by an individual assembly in a given task. In the case of lesion experiments, individuals are assigned a combination of evaluation indexes (for the accomplishment of tasks where the composite model is performing, and the accomplishment of tasks with performance of the eliminated model). For each task, individuals are assigned the maximum of the evaluation indexes achieved by all solutions formed with their membership. Evaluation indexes are further combined to form the fitness value of the individual under discussion.

Just after the testing of collaborator assemblies and the assignment of their fitness values, an evolutionary step is performed on each species to formulate the new generation of its individuals. This process is repeated for a predefined number of evolutionary epochs.

\section{RESULTS}

The effectiveness of the proposed approach is illustrated on the design of a partial brain computational model, which simulates primary motor cortex (M1) - premotor cortex (PMC) interactions. The relevant experiments are indicative of the proposed coevolutionary CNS modelling approach.

PMC (including all non-primary motor areas) is referred as the higher level of motor programming [20], while M1 is considered as the place where the final commands of movement are generated by encoding movement parameters [21]. Thus, PMC activation modulates M1 performance to accomplish goal-driven movements [22]. This organization has been mostly concluded from lesion studies regarding upper limb movement, but similar conclusions are drawn from recent studies aiming at the movement of lower limbs [23], [24].

Computational models of both PMC and M1 have been proposed in the literature eg. [3], [5], [4], which however do not emphasize on their interactions. Recently a simple model of PMC modulating M1 performance is presented in [20], but it is not tested on a real application.

The present work employs the hierarchical collaborative coevolutionary approach to design a model of PMC-M1 realtime performance. In this endeavor, environmental interaction is of utmost importance, since it is difficult to investigate CNS areas performance without embedding the models into a body to interact with its environment. A mobile robot is utilized to support environmental interaction, in order to prove the validity of the result. Specifically, we employ a two wheeled robotic platform equipped with 8 uniformly distributed distance and light sensors.

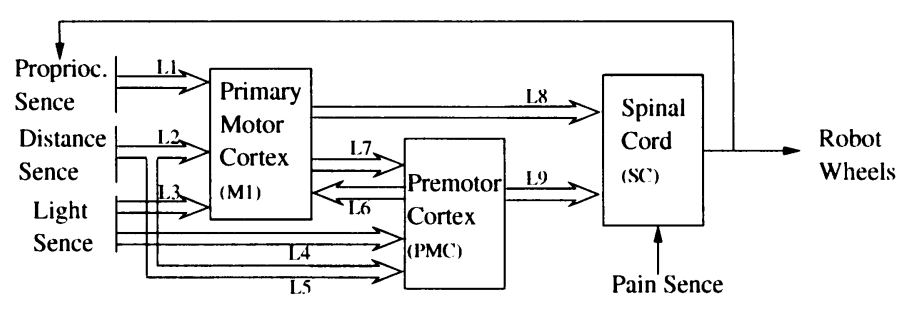

Fig. 4. A schematic overview of the PMC-M1 model. Computational cortical agents are illustrated with blocks, while link agents are illustrated with double arrows.

The experiment aims at reproducing a lesion scenario which is in agreement to the biological data presented above. In accordance to the long-term goal of facilitating artificial organisms with intelligent performance, we emphasize on the accomplishment of behavioral tasks by the robot. The scenario assumes that the performance of the composite PMCM1 model is able to achieve goal-oriented light following robot movement, but when PMC lesion is performed, the goal oriented behavior is affected, and the robot is only able to achieve wall avoidance navigation. By means of the above scenario, the role of each agent in the composite model can be highlighted.

The composite computational model follows the biological prototype [25]. Sensory information is projected to the reciprocally connected neocortical structures via link agents, and from there to the spinal cord with appropriate additional link structures (Fig 4). Pain sense is activated when robot bumps in a wall, and is directly projecting to spinal cord motor neurons to produce a reflexive movement.

The model consists of 12 subcomponents ( 3 cortical and 9 links agents) which have to cooperate to accomplish the desired performance. A hierarchical coevolutionary process is utilized to specify the dynamics of real-time learning in each agent structure. The partial computational structures of the composite model are mapped in a hierarchical coevolutionary tree, as it is illustrated in (Fig 5). Comparing figures 4 and 5, it can be easily realized that hierarchical coevolutionary design does not imply hierarchical performance of the computational model.

One PS species is employed for each partial component of the computational model. Three CG structures are used to drive the hierarchical coevolutionary process. CGl coordinates coevolution of structures relevant to M1 functionality, CG2 coordinates coevolution of structures relevant to PMC functionality, while CG3 coordinates coevolution of groups CG1 and CG2 and also SC structure which is common for both M1 and PMC performance.

PMC lesion is simulated, by deactivating CG2 node, together with all lower level substructures. PMC lesion assumes also the deactivation of all link agent structures under CG2, since their performance has no computational meaning without PMC functioning.

The performance of the PMC-M1 computational model is tested on two different tasks. The composite model is tested 


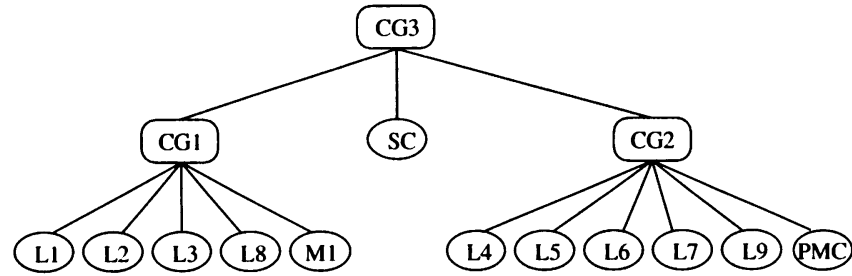

Fig. 5. A schematic overview of the hierarchical coevolutionary process, illustrating the hierarchical mapping of PMC-M1 model.

on the accomplishment of light following behavior, while the eliminated model (after PMC lesion) is tested on wall avoidance navigation. Both behaviors emerge after real-time adjustment of agent structures by means of robot-environment interaction. Separate criteria are designed to evaluate the accomplishment of each task.

The goal-oriented task is simulated by a moving light source that the robot should follow. The success of the task is evaluated by the function:

$$
F_{F}=\left(1 \frac{2 B}{M}\right)^{3} \sum_{M} 2
$$

where we assume that the robot is tested for $M$ steps, is the maximum instant value of all light sensors, and $B$ is the total number of robot bumps. The first term minimizes the number of robot bumps on the walls, while the second supports robot following of the light source.

The success of wall avoidance task is evaluated by the function:

$$
\begin{aligned}
& F_{W}=\left(\sum_{M}\left(\begin{array}{ll}
s+s r & 1
\end{array}\right) *\left(\begin{array}{ll}
1.0 & p^{2}
\end{array}\right)\right) \\
& \text { * }\left(1 \frac{3}{M} \sum_{M} \sqrt{|s \quad s r|}\right)^{3} \\
& \text { * }\left(1 \frac{(s+s r) B}{M}\right)^{3}
\end{aligned}
$$

where we assume that the robot is again tested for $M$ steps, $s, s r$ are the instant speeds of the left and right wheel, $p$ is the maximum instant activation of distance sensors, and $B$ is the total number of robot bumps. The first term seeks for forward movement far from the walls, the second supports straight movement without unreasonable spinning, and the last term minimizes the number of robot bumps on the walls.

These evaluation criteria are used to design fitness functions which guide the coevolutionary process. Specifically, a separate fitness function is designed for each $\mathrm{CG}$ :

$$
\begin{aligned}
& F_{C 1}=F_{W} \sqrt{F_{F}} \\
& F_{C 2}=\sqrt{F_{W} F_{F}} \\
& F_{C} 3=F_{W} F_{F}
\end{aligned}
$$

where $F_{C} \quad{ }_{i}$ represent the fitness function of the $i \quad t h$ CG. All PS species share a common fitness function with the owner CG. It is clear that $F_{C}{ }_{1}$ emphasize more the accomplishment of wall avoidance navigation, $F_{C} 2$ pays more attention on the accomplishment of light following, while $F_{C} 3$ aims to coordinate lower level coevolutionary processes by equally enforcing the accomplishment of both tasks.

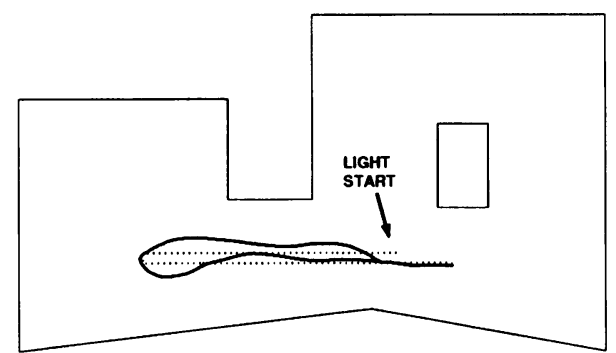

Fig. 6. A sample result of model performance in the light following task by the composite PMC-M1 model. Robot path is illustrated by a solid line, while the dashed line, illustrates the path of light source.

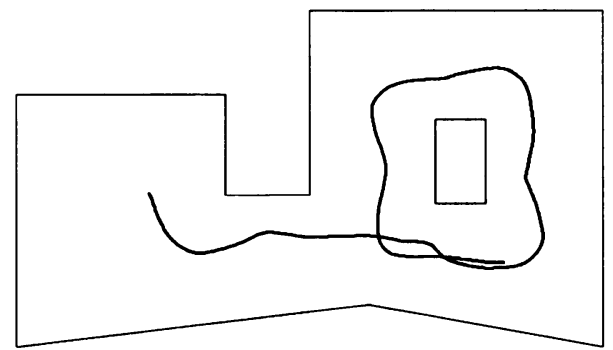

Fig. 7. A sample result of model performance after PMC lesion in the wall avoidance navigation task.

The employment of the above fitness functions enforces the coevolutionary process to consider the hypothetical lesion scenario during the design process. Consequently, partial computational structures are properly designed to develop the desired pre- and post- lesion performance. We mention that even if CG2 structures are not participating in the wall avoidance task, they are also affected by its success in order to avoid exploiting the subset of M1 structures that are unable to accomplish wall avoidance navigation.

Following the above fitness functions different agent structures are designed emphasizing in different criteria of the composite model performance. This fact, together with the successful accomplishment of the lesion scenario highlights the different roles of partial structures in the composite model.

Specifically, M1 is able to move the robot, but without the ability to achieve purposeful motion. The latter is achieved by means of PMC which successfully modulates M1 performance to develop light-following behavior. A sample result of light following behavior from the PMC-M1 model is illustrated in Fig 6. Wall avoidance behavior from the eliminated model is illustrated in Fig 7.

All PS species evolved by populations of 100 individuals. CG1, CG2, and CG3 species are evolved by populations of 200, 200, 300 individuals respectively. Evolution was performed for 70 epochs in synchronous steps for all populations. 


\section{CONCLUSIONS}

In the present work, we introduced a computational framework for the design and implementation of brain models able to replicate biological lesion data. The proposed method is based on the employment of neural agent modules to represent brain areas, which are connected using appropriate link agent structures. The agent-based approach is in accordance to the distributed nature of mammalian CNS. Furthermore, it supports the autonomy of brain areas, and consequently allows the investigation of model performance in lesion conditions.

Agent structures are adjusted in real-time by following a self-organized process which simulates epigenetic learning of biological organisms. The dynamics of epigenetic learning are designed following an evolutionary approach which simulates phylogenesis. As a result, both genetically encoded features and environmental experience specify the performance of the model.

We employ a hierarchical collaborative coevolutionary (HCCE) approach to support design specification of agent structures. The collaborative coevolutionary process is suitable for agents' design because it offers increased search abilities of partial components, and is able to emphasize both the specialty of brain areas and their cooperative performance.

The hierarchical organization of the coevolutionary process supports the elimination of agent structures to simulate lesion experiments. Thus, the role of each partial structure in the composite model can be examined. Additionally, HCCE supplies a mechanism to specify the performance of the model in pre- and post- lesion conditions, by forming appropriate fitness functions. Consequently, the proposed method seems particularly appropriate for implementing reliable models of brain areas, with the ability to replicate lesion data.

Following this approach, the distinct role of each agent structure in the composite model is highlighted. This has been confirmed with the results shown in the previous section, as well as other results obtained in our experiments. (not presented here due to space limitations). Evidently, further work is needed to fully ascertain the general applicability and validity of our approach.

We also note that by adopting the coevolutionary method for design specification, our approach is inherently furnished with the ability to integrate partial brain models. The proposed hierarchical collaborative coevolutionary scheme can be also utilized to integrate the performance of partial brain models, by introducing an appropriate number of additional higher level evolutionary process. Thus, the incremental integration of gradually more partial brain models on top of existing ones constitutes the main direction of our future work. We believe that by exploiting the proposed approach, a powerful method to design large scale reliable brain models can emerge.

\section{REFERENCES}

[1] E. R. Kandel, J. Schwartz, and T. M. Jessell, Principles of Neural Science. Mc Graw Hill, 2000.
[2] R. Cotterill, "Cooperation of the basal ganglia, cerebellum, sensory cerebrum and hippocampus: possible implications for cognition, consciousness, intelligence and creativity," Progress in Neurobiology, vol. 64, no. 1 , pp. 1-33, 2001.

[3] R. Ajemian, D. Bullock, and S. Grossberg, "A model of movement coordinates in motor cortex: posture-dependent changes in the gain and direction of single cell tuning curves." Dep. Cognitive and Neural Systems, Boston University, 2000.

[4] A. Fagg and M. Arbib, "Modeling parietal-premotor interactions in primate control of grasping." Neural Networks, vol. 11, pp. 1277-1303, 1998.

[5] E. Todorov, "Direct cortical control of muscle activation in voluntary arm movements: a model." Nature Neuroscience, vol. 3, pp. 391-398, 2000.

[6] M. Maniadakis and P. Trahanias, "A computational model of neocorticalhippocampal cooperation and its application to self-localization," in Proceedings of 7th European Conference on Artificial Life (ECAL 2003). Springer-Verlag Heidelberg, 2003, pp. 183-190.

[7] R. Aharonov, L. Segev, I. Meilijson, and E. Ruppin, "Localization of function via lesion analysis," Neural Computation, vol. 15, no. 4, pp. 885-913, 2003.

[8] V. Goel, S. Pullara, and J. Grafman, "A computational model of frontal lobe dysfunction: working memory and the tower of hanoi task." Cognitive Science, vol. 25, pp. 287-313, 2001.

[9] T. Polk, P. Simen, R. Lewis, and E. Freedman, "A computational approach to control in complex cognition." Brain Research Interactive, vol. 15, pp. 71-83, 2002.

[10] M. Maniadakis and P. Trahanias, "Modelling brain emergent behaviors through coevolution of neural agents," accepted for publication, Neural Networks, 2005.

[11] _ - "Evolution tunes coevolution: modelling robot cognition mechanisms." in Proc. of Genetic and Evolut. Comput. Conference, (GECCO. 2004), 2004.

[12] E. Rolls and S. Stringer, "On the design of neural networks in the brain by genetic evolution." Progress in Neurobiology, vol. 61, pp. 557-579, 2000.

[13] N. Kasabov and L. Benuskova, "Computational neurogenetics.” Journal of Computational and Theoretical Nanoscience, vol. 1, no. 1, pp. 1-15, 2004.

[14] D. Geary and K. Huffman, "Brain and cognitive evolution: Forms of modularity and functions of mind," Psych. Bulletin, vol. 128, pp. 667698, 2002.

[15] E. Tkaczyk, "Pressure hallucinations and patterns in the brain." Morehead El. Journal of Applicable Mathematics, vol. 1, pp. 1-26, 2001.

[16] D. Floreano and J. Urzelai, "Evolutionary robots with on-line selforganization and behavioral fitness." Neural Networks, vol. 13, pp. 431443, 2000.

[17] M. Poter and K. De Jong, "Cooperative coevolution: An architecture for evolving coadapted subcomponents." Evol. Computation, vol. 8, pp. $1-29,2000$.

[18] M. Delgado, V. F. Zuben, and F. Gomide, "Coevolutionary genetic fuzzy systems: a hierarchical collaborative approach," Fuzzy Sets and Systems, vol. 141, no. 1, pp. 89-106, 2004.

[19] D. Moriarty and R. Miikkulainen, "Forming neural networks through efficient and adaptive coevolution." Evolutionary Computation, vol. 5, no. 4, pp. 373-399, 1997.

[20] S. Kakei, D. Hoffman, and P. Strick, "Sensorimotor transformations in cortical motor areas." Neuroscience Research, vol. 46, pp. 1-10, 2003.

[21] J. Fuster, "Executive frontal functions," Experimental Brain Research, vol. 133 , pp. 66-70, 2000.

[22] J. Kalaska, S. Scott, P. Cisek, and L. Sergio, "Cortical control of reaching movements." Current Opinion in Neurobiology, vol. 7, pp. 849-859, 1997.

[23] I. Miyai, H. Tanabe, I. Sase, H. Eda, I. Oda, I. Konishi, Y. Tsunazawa, T. Suzuki, T. Yanagida, and K. Kubota, "Cortical mapping of gait in humans: a near-infrared spectroscopic topography study." Neuroimage, vol. 14 , no. 5 , pp. 1186-1192, 2001.

[24] C. Sahyoun, A. Floyer-Lea, H. Johansen-Berg, and P. Matthews, "Towards an understanding of gait control: brain activation during the anticipation, preparation, and execution of foot movements." Neurolmage, vol. 21, no. 2, pp. 568-575, 2004.

[25] R. Dum and P. Strick, "Motor areas in the frontal lobe of the primate." Physiology \& Behavior, vol. 77, pp. 677-682, 2002. 